\title{
LA ENSEÑANZA DE LA ANTROPOLOGÍA MÉDICA Y LA SALUD INTERCULTURAL EN MÉXICO: DEL INDIGENISMO CULTURALISTA DEL SIGLO XX A LA INTERCULTURALIDAD EN SALUD DEL SIGLO XXI
}

\author{
Roberto Campos-Navarro',a
}

\begin{abstract}
RESUMEN
El estudio del proceso salud/enfermedad/atención, abordado desde el punto de vista médico y social, es desarrollado de forma especializada por la Antropología Médica. En México esta especialidad tiene sus inicios a finales del siglo XIX. Desde entonces han existido muchas reformas educativas, relacionadas con los propios procesos políticos y el entendimiento y reconocimiento de la pluriculturalidad mexicana, lo que ha permitido ampliar, diversificar y consolidar esta especialidad. En la siguiente se hace una revisión de la evolución histórica de los cursos dedicados a esta materia, los docentes que influyeron en su consolidación, y una presentación sobre el estado actual de los programas académicos que se brindan, destacando la diversidad de otras especialidades en ciencias de la salud que se ven involucradas.
\end{abstract}

Palabras clave: Antropología cultural; Competencia cultural; Diversidad cultural; Educación médica (fuente: DeCS BIREME).

\section{EDUCATION ON MEDICAL ANTHROPOLOGY AND INTERCULTURAL HEALTH IN MEXICO: FROM THE 20 $0^{\text {th }}$ CENTURY CULTURAL INDIGENISM TO THE $21^{\text {ST }}$ CENTURY INTERCULTURALITY}

\begin{abstract}
The study of the health/disease/healthcare process from the socio-medical aspect is the field of the medical anthropology. In Mexico, this medical specialty had its origins at the end of the $19^{\text {th }}$ century. Since then, many educational reforms occurred associated to the political processes and the recognition and better understanding of Mexican pluricultural society; allowing expansion, diversification and consolidation of medical anthropology as an specialty. This review presents the historical evolution of the academic courses on this field, the educators that influenced its consolidation, and the current situation of the available academic programs on medical anthropology. The diversity of specialties from those health sciences that are associated to medical anthropology is emphasized.
\end{abstract}

Key words: Anthropology, cultural; Cultural competency; Cultural diversity; Medical education (source: MeSH NLM).

La formación, adiestramiento y capacitación en el terreno de la antropología médica y la salud intercultural en México, es un tema íntimamente articulado con la emergencia, crecimiento, desarrollo y consolidación de la disciplina antropológica en nuestro país. La docencia se remonta a las actividades aisladas de los primeros antropólogos extranjeros y mexicanos que se dedicaron en forma directa o secundaria al campo del proceso salud/enfermedad/atención (s/e/a).

Si bien se sabe de la presencia de antropólogos extranjeros realizando trabajo de campo en la República Mexicana a finales del siglo XIX y principios del siglo $\mathrm{XX}$, las investigaciones de Carl Lumholtz, Frederick
Starr, Alex Hrdlicka y otros, se concentran en aspectos de la antropología física, en especial la búsqueda de las características raciales propias de los indios mexicanos, con algunas aproximaciones etnográficas y muy escasas referencias al proceso s/e/a, excepto esbozos relacionados con la medicina "tradicional" de los pueblos originarios que visitan. En ningún caso dejaron huella relevante en la enseñanza antropológica.

La antropología -propiamente mexicana- se funda con los trabajos pioneros de Manuel Gamio sobre el mejoramiento de vida de los pobladores del valle de Teotihuacán (1922), donde la alimentación y sus efectos nutricionales ocuparon un importante lugar (1). Gamio

Departamento de Historia y Filosofía de la Medicina, Facultad de Medicina, Universidad Nacional Autónoma de México.

a Médico, especialista en medicina familiar. Maestría y doctorado en Antropología Social. 
representó el primer intento nacionalista por establecer una política pública de incorporación de los pueblos indígenas a la modernidad. Sería el inicio informal del llamado indigenismo como un instrumento del Estado para la integración de los indígenas a la vida nacional.

En Carapan, Michoacán (1932-1933) aparecen los esfuerzos educativos vasconcelianos del maestro Moisés Sáenz, quien propone una antropología social aplicada a la política indigenista de incorporación del indio a la vida nacional. $Y$ tal asimilación cultural se lograría, entre otros programas, mediante la introducción de la educación generalizada y la atención biomédica en pueblos purépechas ${ }^{(1)}$.

Con la creación en 1935 del Instituto Politécnico Nacional (IPN) durante el gobierno revolucionario de Lázaro Cárdenas, se funda dentro de la Escuela de Ciencias Biológicas, el Departamento de Antropología (antecedente de la Escuela Nacional de Antropología e Historia) y la Escuela Superior de Medicina Rural.

A instancias del antropólogo Miguel Othón de Mendizábal se le otorga a esta última escuela, una orientación social, que la diferenciaría de la -hasta esa época monolítica y liberal- Escuela de Medicina dependiente de la Universidad Nacional Autónoma de México (UNAM) ${ }^{(2)}$. Se trataba de un proyecto que intentaba la formación de jóvenes médicos de extracción campesina para que retornaran a sus comunidades para introducir la biomedicina. El propósito fundamental de la Escuela Superior de Medicina Rural era lograr que la distribución de médicos en las zonas rurales resolviera los urgentes problemas de salud pública, disminuyera las enfermedades infecciosas y abatiera los elevados índices de mortalidad materna e infantil.

Los contenidos de la carrera implicaban una formación técnica en aspectos de salubridad general y resolución de problemas médicos cotidianos, con la formación y adiestramiento en sociología y antropología social:

El currículum de la carrera -amén de las materias básicas, técnicas generales y especializadas, comunes- introdujo como modalidad la exigencia de cursos en ciencias sociales. El primero se denominó antropología médica y fue dividido en tres cátedras -antropología física, antropología social y organización social- (...). La inclusión de la antropología física, fue, sin duda, una concesión a la orientación biológica tradicional de la carrera. La antropología social enfocó el estudio de los problemas indígenas y rurales de México, y la organización social, el conocimiento histórico de los pueblos amerindios. Cualesquiera que hayan sido sus defectos, estas cátedras constituyeron una innovación trascendente en el currículum médico ${ }^{(2)}$.

Desde 1938, Alfredo Barrera Vázquez, Julio de la Fuente y Gonzalo Aguirre Beltrán serían los principales docentes de esta novedosa orientación de los estudios médicos de pregrado con fuerte componente social.

En las primeras generaciones saldrían médicos comprometidos con las comunidades indígenas y campesinas ${ }^{(3)}$, por desgracia, este notable esfuerzo no tuvo continuidad porque posteriores egresados prefirieron las comodidades y oportunidades urbanas y en 1965 desaparecería lo rural de la denominación oficial de la escuela.

Demanera simultánea, en 1938nace-con siete profesores y cinco alumnos- la Escuela Nacional de Antropología e Historia (ENAH) como institución educativa, primero perteneciente al IPN, y luego dependiente del Instituto Nacional de Antropología e Historia $\left(\right.$ INAH) ${ }^{(4)}$.

Su misión explícita sería la preparación de los funcionarios que requería la nueva política pública cardenista de incorporar a los indígenas a la vida nacional. El objetivo era ...Estudiar la situación económica y social de los aborígenes, con el fin práctico e inmediato de formular planes concretos de acción, basados en la realidad misma, para obtener su mejoramiento y defender a los indios ante las autoridades federales y locales en todos sus asuntos de interés colectivo ${ }^{(4)}$.

Precisamente con la fundación del Instituto Nacional Indigenista (INI) en 1948, esta institución se convertiría en una de las más importante promotoras y receptoras de etnólogos y antropólogos sociales formados en la ENAH ${ }^{(5)}$. De hecho, la creación de la especialidad de Antropología Social en 1954, se debió a un convenio donde los investigadores y funcionarios del INI daban clases de Antropología Aplicada en la ENAH y las prácticas de campo de los estudiantes se realizaban en los recién creados centros coordinadores del INI en el país. Así fueron maestros Ricardo Pozas, Julio de la Fuente, Alejandro Marroquín y el mismo Gonzalo Aguirre Beltrán ${ }^{(6)}$.

Es así que en las décadas de 1940 y 1950 brillaría la figura del médico y antropólogo Gonzalo Aguirre Beltrán, quien brindaría relevantes aportes teóricos y aplicativos en la antropología social en general (desarrollaría los conceptos de "aculturación" y "regiones de refugio") y de la Antropología Médica en particular. Como discípulo de M. Herskovits y de M. Gamio, sería el intelectual indigenista más reconocido de la corriente culturalista o relativismo cultural en México, y publicaría libros ya 
clásicos de la Antropología Médica como: Los programas de salud en la situación intercultural (1955) ${ }^{(7)}$, Magia y medicina. El proceso de aculturación en la estructura colonial (1963) (2) y "Antropología Médica. Sus desarrollos teóricos en México" (1986) ${ }^{\left({ }^{8}\right)}$. En su amplia trayectoria como médico, investigador docente y funcionario estatal sería (y sigue siendo) punto de referencia de seguidores y contradictores. Pero nada resta méritos a su congruencia teórica/práctica, que lo hizo ser uno de los primeros docentes de antropología médica en el país, director del primer Centro Coordinador TzeltalTzotzil del Instituto Nacional Indigenista en San Cristóbal de Las Casas, Chiapas, director del Instituto Indigenista Interamericano, rector de la Universidad Veracruzana, entre otros cargos ${ }^{(9)}$. No hace falta recordar que fue -como ya apuntamos en líneas previas- pionero de la enseñanza de la Antropología Médica en la carrera de medicina del IPN y de los epidemiólogos y salubristas egresados de la Escuela de Salud Pública en la ciudad de México, cuyo curso se hizo celebre en los inicios de la década de $1950{ }^{(10)}$.

Cabe mencionar la participación de antropólogos norteamericanos dedicados directa e indirectamente en la Antropología Aplicada en programas de salud en América Latina y que influyeron con sus clases o sus obras escritas en la formación de discípulos en México. El primero de ellos sería Robert Redfield de la Universidad de Chicago. A finales de la década de 1920, Redfield hace sus primeras investigaciones etnográficas en México, describiendo las relaciones entre magia, religión y medicina en los pobladores nahuas de Tepoztlán, Morelos. Años más tarde, con su experiencia de campo en Yucatán surgirá su conocida teoría del continuum folk-urbano y el desarrollo de comunidad e influiría en la formación de los antropólogos sociales de la década de 1950 y 1960 a través de Fernando Cámara, uno de sus principales discípulos en México ${ }^{(11)}$.

Más adelante George Foster, de la Universidad de California, enviado a la ENAH por el Smithsonian Institution para la preparación de investigadores locales, autor de Las culturas tradicionales y los cambios técnicos (1964) ${ }^{(12)}$, Antropología aplicada (1974) ${ }^{(13)}$, textos donde aparecen innumerables ejemplos de procesos inducidos de cambio, relacionados con la salud y la medicina, partiendo de la consideración de que la antropología aplicada ...Se ocupa en gran medida del cambio social y cultural que se presenta en el desarrollo y modernización del mundo contemporáneo ${ }^{(13)}$ Años más tarde escribiría con Barbara G. Anderson Medical Anthropology (1978) ${ }^{(14)}$ aún sin edición en español.

De la Universidad del estado de Michigan, el Dr. Arthur Rubel, autor principal de la investigación sobre el síndrome del susto en México e Hispanoamérica, dictó conferencias y talleres en México, en especial con el Seminario de Medicina del Hombre en su Totalidad, del Hospital General de México, dirigido por el Dr. Fernando Martínez Cortés ${ }^{(15)}$. Este seminario reunió a una pléyade de médicos y antropólogos que influirían en escuelas de medicina y de Antropología. Algunos de los connotados participantes fueron Gonzalo Aguirre Beltrán, Alfredo López Austin, Luis Alberto Vargas, Carlos Viesca, Noemí Quesada, Isabel Lagarriga, Silvia Ortiz, Carmen Anzúrez y Leticia Casillas ${ }^{(16)}$.

Otra profesora contratada por la Institución Smithsoniana sería Isabel Kelly, quien realizaría trabajo de campo en Veracruz y la región lagunera compartida por Durango y Coahuila, autora de varias monografías relativas a la medicina tradicional mexicana y profesora de varios médicos y antropólogos inmiscuidos en la salubridad pública como Héctor García-Manzanedo.

Con el movimiento del 68 surge en la ENAH la corriente crítica al indigenismo oficial, sobre todo el que representa Aguirre Beltrán, y a los programas de Antropología Aplicada generados por la corriente culturalista norteamericana. De este modo, la enseñanza de la Antropología Médica se estanca, y tan sólo se brindan en la licenciatura algunos cursos aislados y discontinuos relacionados con la Etnomedicina y la Medicina Tradicional. Cabe recordar que en esta época el Instituto Nacional de Antropología e Historia creó el Departamento de Estudios en Antropología Social (DEAS) donde Isabel Lagarriga, Silvia Ortiz Echániz y otros investigadores han dedicado sus esfuerzos académicos en la formación institucional de nuevas generaciones, siendo la Antropología Médica, una de sus áreas de trabajo.

A principios de la década de 1980 se crea en la ENAH el posgrado en Antropología Social con talleres específicos sobre cuestiones agrarias, obreras, étnicas, cultura e ideología, añadiéndose más tarde un quinto taller, el de Antropología Médica. El taller es creado a iniciativa de E. Menéndez y el apoyo del autor como profesor adjunto, Si bien E. Menéndez estaba como investigador en el CIESAS, en el posgrado de la ENAH descarga sus habilidades docentes en la formación de antropólogos médicos. Desde 1985 y hasta el año 2000 pasarían múltiples generaciones de maestría, requiriéndose desde 1996 la ampliación a estudios de doctorado.

Con una orientación fundamentada en la antropología médica crítica, los cursos bianuales pretendieron y establecieron una concepción del proceso salud/ enfermedad/atención más allá de sus componentes netamente culturalistas, para introducir modelos 
explicativos de carácter sociopolítico. Al apartarse del relativismo cultural dominante se inicia un enfoque diferente. Durante el primer año se describe y analiza en forma crítica los modelos antropológicos explicativos vigentes del proceso s/e/a. Se ofrece nociones sobre demografía, epidemiología y estructura de los servicios de salud, incluidos los que brinda la biomedicina, las medicinas subalternas y la autoatención.

Más adelante, se profundiza en la teoría de los modelos médicos, que desarrolla $\mathrm{E}$. Menéndez desde la década de 1970 y que plasma en obras importantes como: Poder, estratificación y salud. Análisis de las condiciones sociales y económicas de la enfermedad en Yucatán (1981) $^{(18)}$, Morir de alcohol. Saber y hegemonía médica (1990) ${ }^{(19)}$ y La parte negada de la cultura. Relativismo, diferencias y racismo (2002) ${ }^{(20)}$. Igualmente en el taller de antropología médica se discute y polemiza sobre el concepto de normal/patológico, las teorías de la desviación mental y la epidemiología socio-cultural.

Los alumnos que egresan realizaron sus tesis con temas como salud ocupacional, estrategias urbanas ante las enfermedades, los saberes y las prácticas médicas maternas, el problema de la vacunación en poblaciones urbanas marginales, las estrategias de atención médica en desastres por volcanes, las prácticas curanderiles urbanas, la diabetes mellitus y SIDA en ciudades mexicanas, los movimientos sociales de autogestión en salud, los programas rurales para prevenir la desnutrición, las concepciones de la sexualidad rural, los procesos de autoatención curativa en poblaciones zapatistas de Chiapas, entre otras.

En forma lamentable, y debido a diferencias administrativas que quisieron imponerse sobre los aspectos académicos, los tres maestros que dirigían el taller de maestría y doctorado ( $E$. Menéndez, M.E. Módena y R. Campos-Navarro) tuvieron que renunciar en el año 2000 con un carácter irrevocable.

En la misma Ciudad de México, a nivel de posgrado en 1973 se crea el Centro de Investigaciones Superiores del Instituto Nacional de Antropología e Historia (CISINAH) que luego en 1980 se transformaría en el Centro de Investigaciones y Estudios Superiores en Antropología Social (CIESAS), una institución autónoma dependiente de la Secretaría de Educación Pública (21). En ella, surgirían en la década de 1990 los estudios de maestría y doctorado en Antropología Social con línea de investigación en Antropología Médica (22), de donde han egresado múltiples generaciones, y en la actualidad existen además cursos regulares de antropología médica en los planteles ubicados en la ciudad de Oaxaca y en San Cristóbal de Las Casas, Chiapas. Vale mencionar profesores como el mismo Eduardo Menéndez, María Eugenia Módena, Rosa María Osorio, Sergio Lerín, Paola Sesia, Graciela Freyermuth.

Al igual que la ENAH la orientación corresponde a la corriente denominada como Antropología Médica crítica. Entre los principales contenidos de la maestría del CIESAS destacan: el campo de la Antropología Médica, las perspectivas analíticas del proceso salud/ enfermedad/atención, el pluralismo médico con modelos, sistemas y saberes médicos, la conducta de búsqueda de atención (carrera del enfermo, trayectorias de atención, el uso de los recursos de atención, etc.) y la estructura de la atención curativa en México. En una segunda parte se analiza las categorías teóricas relativas a los procesos binarios de salud/enfermedad, normal/ patológico, normal/anormal, enfermedad/padecimiento y biológico/cultural. Las teorías sobre la desviación social, la construcción social, cultural, económica y política de la vejez, el género, la maternidad y el alcoholismo. El proceso salud-enfermedad-atención como estructura social y de significado, donde se describe y analiza los temas de estratificación social, estratificación étnica, la dimensión intercultural; la Economía Política y el proceso salud-enfermedad-atención. La desigualdad en contexto indígena. Saberes, representaciones y prácticas. El cuerpo y el dolor como representaciones y prácticas. La enfermedad y la salud como metáforas. La enfermedad como identidad y estigma. Los padecimientos mentales y la enfermedad como experiencia y estilo de vida.

En el doctorado en Antropología con línea de especialización en Antropología Médica que se inició en el 2007, se pretende una profundización de los contenidos de la maestría pero enfocados al apoyo de las investigaciones de los doctorantes.

Sin duda, la influencia de E. Ménéndez como formador de profesionistas tanto en la ENAH, como en el CIESAS y otras instancias académicas, ha dejado una profunda huella pues como afirman G. Freyermuth y P. Sesia: Sus alumnos ya son líderes de grupos y están insertos en universidades, centros de investigación, organismos no gubernamentales y en la administración pública (23). En este sentido, se han creado maestrías y diplomados con líneas de investigación en Antropología Médica en CIESAS-Sureste (San Cristóbal de Las Casas, Chiapas) y CIESAS-Istmo (con sede en Oaxaca, Oax) con el apoyo académico del Seminario Permanente de Antropología Médica que se efectúa con regularidad en la capital del país.

En parte, influidos por la trayectoria de E. Menéndez en España y por otra, la solidez académica de su posgrado, la Universitat Rovira i Virgili, ubicada en Tarragona daría 
albergue a múltiples estudiantes de maestría y doctorado en Antropología Médica, y que se reincorporarían al CIESAS y otras instituciones nacionales de prestigio.

Por otra parte, dentro de la formación biomédica, la Facultad de Medicina de la Universidad Nacional Autónoma de México (UNAM), estableció en 1975 un programa piloto de educación médica de pregrado denominado A-36 que incluyó docencia antropológica a estudiantes y profesores ${ }^{(24)}$. Pero no fue sino hasta 1985 que con la promoción y estímulo del Dr. Carlos Viesca Treviño -activo participante del Seminario del Hombre en su Totalidad- se planteó un programa curricular de pregrado que incluyó la historia de la medicina, y dentro de ella -por primera vez- se abarcó en forma obligatoria el área de antropología médica. Se consideró que en la formación del estudiante de medicina se debía lograr una mayor aproximación al enfoque humanístico y, por lo tanto, dentro de la asignatura de Historia y Filosofía de la Medicina se debió compartir espacios teóricos y de investigación con la Ética y la Antropología de la Medicina.

Dada la magnitud de la matrícula estudiantil, donde la Facultad recibe en licenciatura un promedio de 800 a 900 alumnos por año, se tuvo que contratar profesoresmédicos que tuvieran estudios previos de Antropología, o bien, antropólogos que tuvieran experiencia alrededor del proceso salud/enfermedad/atención.

En la actualidad, el grupo colegiado de profesores ha realizado modificaciones pertinentes al curso, que permiten que el estudiante tenga una mayor cercanía a los problemas más directos de la población rural y urbana del país. En forma explícita se determina que el propósito del curso de Antropología Médica no es el de transformar a los médicos en antropólogos ni mucho menos en curanderos ${ }^{(25)}$.

El temario teórico incluye cuatro bloques. En el primero se delinean los objetivos de la Antropología, sus ramas y subdisciplinas, el lugar que corresponde a la Antropología Médica, su campo de estudio, sus instrumentos y metodología de trabajo. En el segundo se considera el sistema de creencias y su concreción en el mundo mesoamericano. En el tercero se aborda la teoría de $\mathrm{E}$. Menéndez sobre los modelos médicos, las características estructurales y coyunturales del modelo médico hegemónico, del modelo de autoatención y el modelo médico alternativo subordinado. Se particulariza acerca de la medicina popular-tradicional mexicana en cuanto a sus concepciones y cosmovisiones, sus recursos humanos y materiales, sus procedimientos curativos y los tratamientos herbolarios y rituales más frecuentes, así como un acercamiento a la epidemiología socio-cultural y a los síndromes culturalmente delimitados o dependientes de la cultura. En el tercer bloque se analiza el proceso salud/enfermedad/atención como un fenómeno social, su relación con lo normal y lo patológico. Los procesos bioculturales de embarazo, parto, puerperio, alimentación, vejez y muerte. En el cuarto bloque se analiza la relación médico-paciente, sus características sociales y antropológicas, la proxemia, los procesos de salud y práctica médica intercultural $y$, para finalizar, una sesión sobre la concepción del hombre en su totalidad. Hasta ahora su principal falencia es la ausencia de prácticas de campo que muestren la pertinencia de los temas teóricos y, por ende, la utilidad de la asignatura.

Con la aprobación del nuevo plan de estudios 2010 de la Facultad de Medicina, el curso denominado "Antropología médica e interculturalidad" se separa de la materia de historia de la medicina y de la ética médica, se transforma en una asignatura independiente que se abordará en el tercer año de la carrera de Medicina. Sin duda un logro trascendente desde el punto de vista disciplinar pero también un reto para ofrecer una asignatura de corta duración (alrededor de 40 horas) que mantenga contenidos teóricos fundamentales pero que también ofrezca una perspectiva práctica con fines aplicativos.

Con un temario similar pero sintetizado, y por iniciativa del autor, desde 1997 se ha realizado un diplomado anual de Antropología Médica con presencia de estudiantes de Medicina, Enfermería, Odontología, Antropología y otras carreras. Esta iniciativa surge ante la ausencia de estudios específicos de maestría en Antropología Médica desde el área biomédica (en concreto en nuestra Facultad de Medicina se viene negociando desde hace varios lustros) y ante la demanda creciente de un curso introductorio de posgrado que no signifique los tiempos a largo plazo que requieren las maestrías de las instituciones antropológicas.

En el diplomado participan los profesores del Departamento, y además han sido invitados antropólogos, médicos y etnobotánicos de reconocido prestigio nacional e internacional como E. Menéndez, Carlos Zolla, Fernando Martínez Cortés, Abigaíl Aguilar, Arnoldo Kraus, Montserrat Salas, Rosa María Osorio, entre otros. Este diplomado ha sido dirigido por el autor, luego Zuanilda Mendoza / Elia Nora Arganis y ahora por Alfredo Paulo Maya / Adriana Ruiz Llanos.

Durante el periodo comprendido entre abril de 2001 y marzo de 2002, con el apoyo financiero de la cooperación Italiana y otras instituciones internacionales, la Facultad de Medicina de la UNAM (secundada por su notable 
experiencia docente en el campo antropológico) realizó un primer diplomado de salud intercultural en coordinación con la Universidad Autónoma Tomás Frías de la ciudad boliviana de Potosí. Este diplomado se distinguió por su carácter teórico y práctico donde los alumnos se graduaron con la presentación pública de sus trabajos de investigación-acción.

En dicho diplomado se incluyó diez módulos interrelacionados. En el primero y dada la heterogeneidad de los participantes (médicos, enfermeras, trabajadores sociales, funcionarios de hospitales, profesores universitarios, e incluso, una arquitecta) se introdujeron las herramientas e instrumentos esenciales de la investigación social cualitativa que condujeran a la formulación de un proyecto de investigación-acción. En el segundo módulo, se presentaron las experiencias contemporáneas más representativas de salud intercultural en América Latina. En el tercero, el concepto de cultura, sistemas y modelos médicos, medicina tradicional indígena y popular. En el cuarto las bases nosológicas y prácticas de la medicina indígena potosina con explicación de los conceptos de cosmovisión, pensamiento mágico-simbólico, etno-clasificación, y los elementos de aproximación y conflicto con la medicina occidental. En el quinto el tema de la interculturalidad y acceso a los servicios de salud, detallándose conceptos como multiculturalidad, Estado, nación, autonomía, administración estatal e interculturalidad. En el sexto se abordó la farmacopea indígena y remedios herbolarios, con una práctica denominada "caminata botánica" que sirvió para el reconocimiento, recolección, nomenclatura y usos de las principales plantas medicinales de la región. En el séptimo se profundizó sobre la relación médicopaciente, los principales conflictos, la comunicación en contextos interculturales, técnicas para mejorarla, humanización de dicha relación médico-paciente, etc. En el octavo se estudió la epidemiología sociocultural, abordándose las dimensiones sociales y culturales de la enfermedad, los conceptos de s/e/a en zonas indígenas, los síndromes de culturalmente delimitados. En el noveno se estudió el enfoque intercultural en el desarrollo de los programas de educación para la salud, sobre todo el manejo de los conceptos de información, educación y comunicación en el mundo quechua. Finalmente en el décimo fue abordado el tema de la dieta y la nutrición en términos interculturales. Cabe mencionar que se establecieron dos módulos adicionales para apoyar los trabajos de investigación de los estudiantes ${ }^{(25)}$.

En este primer diplomado participaron profesores bolivianos y extranjeros de primera línea como Xavier Albó, Jaime Zalles, Oscar Velasco, Iván Castellón, Gerardo Fernández, Andrea Caprara, Margarita Sáez, Jaime Ibacache, Abigaíl Aguilar, Bruno Parodi, Adriana
Ruiz, Luis Alberto Vargas y otros, bajo la coordinación general de Luca Citarella y R. Campos-Navarro.

Un segundo diplomado potosino, y luego una par de réplicas del diplomado se harían en Ayacucho, Perú con la participación de la Universidad Mayor de San Cristóbal de Huamanga y el apoyo logístico y financiero de una agencia española llamada AMARES. El temario sería similar con algunas leves modificaciones.

En México se crea en el año 2001 la Dirección de Medicina Tradicional y Desarrollo Intercultural (26) en la estructura de la Secretaría (o ministerio) de Salud y en los años 2003 y 2004 se promueve mediante una ONG denominada Yolpahtli (mayormente conformada por académicos del Departamento de Historia y Filosofía de la Medicina, y encabezada por el autor) la capacitación de funcionarios de la Secretaría de Salud en los estados con mayor población indígena en el país. Así se realizarían cursos de sensibilización y capacitación en el occidente (Morelia y Tepic), en el sur (Campeche y Puebla) y un par de ellos en la Ciudad de México. El objetivo era que estos cursos fueran replicados en el resto de la República Mexicana.

En años posteriores se multiplicarían los cursos por todo el país, en especial Veracruz, Oaxaca y Yucatán. Por ejemplo, en Veracruz, la Secretaría de Salud local crea el Programa de Salud Indígena y Medicina Tradicional con Enfoque Intercultural y ofreció tres cursos en forma de taller donde asiste personal de salud, sobre todo, médicos pasantes en servicio social, enfermeras, promotores de salud y trabajadores sociales ${ }^{(27)}$. En Oaxaca es retomado el tema por ONG que sensibilizan al personal de salud de las instituciones oficiales (Grupo de estudios sobre la mujer "Rosario Castellanos A.C.") (28), y en Yucatán por la Secretaría de Salud del estado, junto con la Universidad Autónoma de Yucatán (29).

En cuanto a maestrías y doctorados relacionados con la antropología médica y la interculturalidad, en la UNAM se encuentra el Instituto de Investigaciones Antropológicas (IIA-UNAM), que si bien no ofrece estudios específicos De Antropología Médica, sí brinda cursos al respecto como Antropología del Cuerpo, Etnología de la Salud-Enfermedad, Antropología de la Medicina, Antropología y Alimentación y otros similares, que dan apoyo teórico para aquellos estudiantes que manejan protocolos de investigación que se encuentran dentro del campo temático de la Antropología Médica. Dependiendo del IIA-UNAM se tiene el Programa de Investigaciones Multidisciplinarias sobre Mesoamérica y el Sureste (PROIMSSE) que ha desarrollado una línea de investigación y docencia sobre medicina tzeltal y sobre las representaciones sociales de la diabetes 
mellitus en pobladores maya-hablantes de los Altos de Chiapas.

Otro espacio sería el posgrado de Ciencias de la Salud de la propia Facultad de Medicina de la UNAM que tiene un área de "Sistemas de salud" donde se desarrollan algunas clases de antropología de la medicina.

A finales del 2004 en la misma UNAM se crea el Programa Universitario México, Nación Multicultural, con el propósito de dar respuesta a ...los procesos generados por la notable expansión de las temáticas de la multiculturalidad, la interculturalidad, las relaciones interétnicas y el reconocimiento de la diversidad y pluralidad social y natural de México, América y el mundo (...) la universidad aparece, así como, la institución más idónea para impulsar sistemáticamente el desarrollo de nuevos campos del saber, de la formación de recursos humanos, el análisis de las políticas y los proyectos culturales, la significación de los movimientos etnopolíticos y el conocimiento, preservación y desarrollo de los patrimonios culturales ${ }^{(30)}$.

Este programa, entre otros desafíos, ha emprendido proyectos de recopilación informativa, investigación, difusión y en lo que nos interesa, la docencia. El proyecto docente incluye la materia optativa México, Nación Multicultural, que se ofrece a todas las escuelas y facultades de la UNAM y otras de México (Chiapas, Tabasco) y el extranjero (Texas y República Dominicana), y entre otras temáticas se ofrece el módulo Salud y Medicina entre los Pueblos Indígenas. Además vale mencionar que ha presentado en forma reciente, su Biblioteca Digital de la Medicina Tradicional Mexicana, que constituye un aporte sumamente valioso para los estudiosos de los recursos humanos, materiales y simbólicos de la medicina tradicional y que se encuentra en: www.medicinatradicionalmexicana.unam.mx

Desde 1997 la Escuela Superior de Medicina del IPN sin la adjetivización de "rural" introdujo en el tercer semestre, la asignatura específica de Antropología Médica (31) y, en la actualidad, brinda cinco unidades: Introducción a la antropología (12 horas), métodos y técnicas antropológicas (8 horas), la construcción sociocultural de la enfermedad (16 horas y donde se incluye el tema de medicina intercultural), procesos bioculturales (14 horas) y relación médico-paciente (12 horas). En total 62 horas teóricas que se complementan con 40 horas de prácticas escolares obligatorias ${ }^{(32)}$. Quizá este sea uno de los mejores programas de Antropología Médica dirigido a estudiantes de pregrado en Medicina, en función de los contenidos temáticos, el número suficiente de horas, la actualización constante, las prácticas de campo, y su única desventaja sería que se brinda en los primeros semestres del año, cuando lo óptimo es hacerlo antes del internado hospitalario y la práctica del servicio social, cuando los alumnos ya tienen un mayor contacto con los pacientes y sus familiares.

También dependiente del IPN, la Escuela Nacional de Medicina y Homeopatía ha logrado la inclusión de la Antropología Médica en el cuarto semestre, con articulación a la historia de la medicina, y un declarado énfasis en la medicina tradicional ${ }^{(33)}$.

Ninguna escuela y facultad médica de origen privado ha dado la asignatura, excepto la de la Universidad Latinoamericana, una pequeña institución escolar de reciente creación ubicada en Cuernavaca, Morelos, que mantiene dos seminarios; uno de medicina tradicional y otro de Antropología Médica con muchos contenidos procedentes de la UNAM.

Sinqueexistaun programaparticularizadodeantropología médica y salud intercultural tenemos conocimiento de cursos y seminarios en la Escuela de Salud Pública (ahora Instituto Nacional de Salud Pública), el Centro Regional Morelos del INAH (con estudios centrados en los agentes de la medicina tradicional), la Universidad de Guadalajara (con profundización en la investigación cualitativa en salud), el Colegio de Michoacán (con múltiples tesis sobre medicina purépecha y estudios de salud reproductiva), El Colegio de Sonora (y sus interesantes aportes en epidemiología sociocultural), la Universidad Veracruzana (en sus escuelas de antropología y medicina), la Universidad Autónoma del Estado de México, la Universidad de las Américas en Puebla, el Colegio de la Frontera Sur (ECOSUR) y en varias escuelas de escuelas de enfermería (donde a veces reducen la antropología médica a la práctica y saberes de la medicina tradicional mexicana) ${ }^{(34)}$.

Podemos concluir afirmando que la enseñanza contemporánea de la antropología médica a nivel de posgrado se consigue mejor mediante las escuelas de Antropología como CIESAS y el Instituto de Investigaciones Antropológicas de la UNAM pero si se trata de la enseñanza de pregrado en el campo biomédico, la Escuela Superior de Medicina del IPN y la Facultad de Medicina de la UNAM, en la actualidad son las más idóneas.

Sin duda, laAntropología Médica en México se encuentra en fase de consolidación y expansión. Su enseñanza se multiplicará en forma paralela, pues la demanda de profesores a todos niveles es y seguirá creciente. Licenciaturas en Antropología, Medicina, Nutrición, Enfermería, Salud Pública, Trabajo Social y otras, así lo requieren. Diplomados, maestrías y doctorados con 
especialización en Antropología Médica se encuentran en pleno desarrollo. El fenómeno internacional de globalización no homogeneizante, de reconocimiento a la pluriculturalidad y la interculturalidad, la reciente afirmación de los derechos de los pueblos indígenas (y sus demandas centenarias), el pluralismo de modelos y sistemas médicos, entre otros procesos, han conducido a esta expansión de la Antropología, y en especial de la orientación médica. Con este panorama, el compromiso académico y la responsabilidad educativa en el campo de la antropología médica mexicana resultan ineludibles.

\section{Conflictos de Interés}

El autor declara no tener conflictos de interés en la publicación de este artículo.

\section{REFERENCIAS BIBLIOGRÁFICAS}

1. Comas J. La antropología social aplicada en México: trayectoria y antología. México DF: Instituto Indigenista Interamericano; 1976.

2. Aguirre-Beltrán G. Magia y medicina. El proceso de aculturación en la estructura colonial. México DF: Instituto Nacional Indigenista; 1963.

3. Arganis EN. La antropología en la formación del médico rural (1938-1965). X Congreso Nacional y V Internacional de Historia y Filosofía de la Medicina. Morelia: Sociedad Mexicana de Historia y Filosofía de la Medicina; 2010.

4. Cámara F. Aportaciones de los antropólogos mexicanos formados en la década de los cuarenta. En: Olivera M, editor. Cuatro décadas de la Escuela Nacional de Antropología e Historia. México DF: Escuela Nacional de Antropología e Historia; 1982.

5. Valencia E. Aportaciones de los antropólogos mexicanos formados en la década de los cuarenta. En: Olivera M, editor. Cuatro décadas de la Escuela Nacional de Antropología e Historia. México: Escuela Nacional de Antropología e Historia; 1982.

6. Bonfil G. La generación de los magníficos y el resultado de las posiciones críticas en Antropología. En: Olivera $M$, editor. Cuatro décadas de la Escuela Nacional de Antropología e Historia. México: Escuela Nacional de Antropología e Historia; 1982.

7. Aguirre-Beltrán G. Programas de salud en la situación intercultural. México: Fondo de Cultura Económica; 1955.

8. Aguirre-Beltrán G. Antropología Médica. Sus desarrollos teóricos en México. México: Fondo de Cultura Económica; 1990.

9. Báez-Jorge F (coord.). Memorial crítico: diálogos con la obra de Gonzalo Aguirre Beltrán en el centenario de su natalicio. México: Gobierno del Estado de Veracruz; 2008.

10. Zolla C. Antropología médica, salud y medicina en la obra de Gonzalo Aguirre Beltrán. En: Baez-Jorge F. (coord.) Memorial crítico: diálogos con la obra de Gonzalo Aguirre Beltrán en el centenario de su natalicio. Xalapa: Gobierno del Estado de Veracruz; 2008.
11. Bonfil G. La generación de los magníficos y el resultado de las posiciones críticas en antropología. En: Olivera M, editor. Cuatro décadas de la Escuela Nacional de Antropología e Historia. México DF: Escuela Nacional de Antropología e Historia; 1982.

12. Foster G. Las culturas tradicionales y los cambios técnicos. México DF: Fondo de Cultura Económica; 1964.

13. Foster G. Antropología aplicada. México DF: Fondo de Cultura Económica; 1974.

14. Foster G, Anderson B. Medical anthropology. New York: John Wiley and Sons; 1978.

15. Rubel A, O’Nell C, Collado R. Susto, una enfermedad popular. México DF: Fondo de Cultura Económica; 1989.

16. Lagarriga I. La posesión demoníaca: una expresión popular de la de la concepción de la enfermedad mental. En: BaezJorge F. (coord.) Memorial crítico: diálogos con la obra de Gonzalo Aguirre Beltrán en el centenario de su natalicio. Xalapa: Gobierno del Estado de Veracruz; 2008.

17. Menéndez E. Poder, estratificación y salud: Análisis de las condiciones sociales y económicas de la enfermedad en Yucatán. México: Centro de Investigaciones y Estudios Superiores en Antropología Social; 1981.

18. Menéndez E. Morir de alcohol. Saber y hegemonía médica. México DF: Patria; 1990.

19. Menéndez E. La parte negada de la cultura. Relativismo, diferencias y racismo. Barcelona: Ediciones Bellaterra; 2002.

20. García-Acosta V. Gonzalo Aguirre Beltrán, creación y descentralización del CIESAS. En: Baez-Jorge F. (coord.) Memorial crítico: diálogos con la obra de Gonzalo Aguirre Beltrán en el centenario de su natalicio. Xalapa: Gobierno del Estado de Veracruz; 2008.

21. Escuela Nacional de Antropología e Historia. Maestrías en antropología social, historia-etnohistoria y lingüística. Plan de estudios. México DF: Escuela Nacional de Antropología e Historia; 1988.

22. Freyermuth G, Sesia P. Del curanderismo a la influenza aviaria: viejas y nuevas perspectivas de la antropología médica. Desacatos. 2006; 20: 9-28.

23. Hafer F. Plan para la educación médica al servicio de pueblo mexicano. América Indígena. 1977; 37(2): 425-34.

24. Campos-Navarro R. La enseñanza de la medicina populartradicional en la Facultad de Medicina de la Universidad Nacional Autónoma de México. Kallawaya. 1999; 6: 7-18.

25. Campos-Navarro R, Citarella L. Willaqkuna. Un programa en salud intercultural en Bolivia. En: Fernández-Juárez, Gerardo (coord.). Salud e interculturalidad en América Latina. Perspectivas antropológicas. Quito: Abya Yala / Agencia Bolhispana/ Universidad de Castilla La Mancha; 2004.

26. Secretaría de Salud, Dirección de Medicina Tradicional y Desarrollo Intercultural. Fortalecimiento y desarrollo de la medicina tradicional mexicana y su relación intercultural con la medicina institucional. Mexico DF: Secretaría de Salud; 2003.

27. Rodríguez-Hernández A, Barrera I, Vázquez-Aguilar K, Cortés E. Medicina tradicional en la Huasteca y el Totonacapan. Socialización de una experiencia de intervención institucional con enfoque intercultural en el Estado de Veracruz. Xalapa: Servicios de Salud de Veracruz; 2007. 
28. Varios. Salud e interculturalidad. Oaxaca: Grupo de estudios sobre la mujer "Rosario Castellanos, A.C."; 2006.

29. Quattrocchi P, Güémez-Pineda $M$, (coords.). Salud reproductiva e interculturalidad en el Yucatán de hoy. Mérida: Asociación de Investigaciones Etno-antropológicas y Sociales de Trieste / Unidad de Ciencias Sociales del Centro de Investigaciones Regionales Dr. Hideyo Noguchi de la UADY / Unidad Académica de Ciencias Sociales y Humanidades de la UNAM / Instituto para el desarrollo de la cultura Maya del Estado de Yucatán / Comisión Nacional para el Desarrollo de los Pueblos Indígenas; 2007.

30. Universidad Nacional Autónoma de México. Programa Universitario México, Nación Multicultural. México DF: PUMC, UNAM; 2005.

31. Cruz-Santacruz R, Ruiz-Belman A. La enseñanza de la antropología médica en la Escuela Superior de Medicina del Instituto Politécnico Nacional. En; Izquierdo $\mathrm{T}$, Aguirre $\mathrm{E}$, editores. Medicina tradicional en el contexto de la Antropología Médica. Experiencias que iluminan la enseñanza y la práctica en las Ciencias de la Salud. México DF: Universidad Autónoma Metropolitana; 2006.

32. Instituto Politécnico Nacional, Escuela Superior de Medicina. Temario general del curso de antropología médica (enero-junio 2010). México DF: Instituto Politécnico Nacional; 2010.

33. Camacho R, Guzmán C. Constructivismo y medicina tradicional. (Una experiencia en la formación médica). En: Izquierdo T, Aguirre E, editores. Medicina tradicional en el contexto de la antropología médica. Experiencias que iluminan la enseñanza y la práctica en las ciencias de la salud. México: Universidad Autónoma Metropolitana; 2006.

34. Martínez-Camilo N. La enseñanza de la terapéutica tradicional mexicana en la Escuela Superior de Enfermería y Obstetricia del Instituto Politécnico Nacional. En; Izquierdo $\mathrm{T}$, Aguirre E, editores. Medicina tradicional en el contexto de la Antropología Médica. Experiencias que iluminan la enseñanza y la práctica en las Ciencias de la Salud. México: Universidad Autónoma Metropolitana; 2006.

Correspondencia: Roberto Campos Navarro

Dirección: Brasil 33 Centro Histórico, C.P. 04060, Distrito Federal, México.

Correo electrónico: rcampos@unam.mx

\section{Consulte las ediciones anteriores de la Revista Peruana de Medicina Experimental y Salud Pública en}

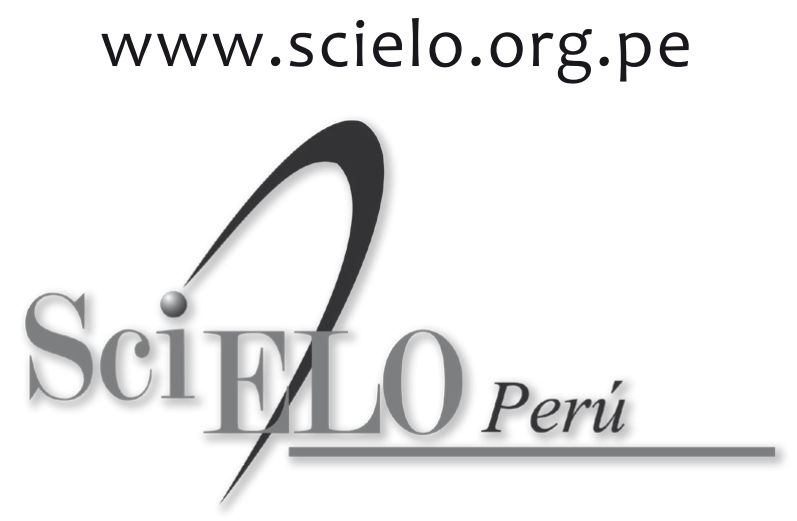

